\title{
DISCOVERY OF POINT SOURCES IN THE HELMHOLTZ EQUATION POSED IN UNKNOWN DOMAINS WITH OBSTACLES*
}

\author{
YANINA LANDA ${ }^{\dagger}$, NICOLAY M. TANUSHEV ${ }^{\ddagger}$, AND RICHARD TSAI ${ }^{\S}$
}

\begin{abstract}
We consider an inverse source problem for the Helmholtz equation in domains with possibly unknown obstacles, which are much larger than the wavelength. The inverse problem is to determine the number and locations of point sources in the domain based on sparse measurements of the wave field. Our proposed strategy relies on solving a local inverse scattering problem to obtain the incoming directions of waves passing through an observation location. We formulate the problem as an $L^{1}$ constrained minimization problem and solve it using the Bregman iterative procedure. The wave direction rays are then traced back and sources are uniquely determined at the intersection of the rays from several observing locations. We present examples in $2 \mathrm{D}$, however all of the formulas and methods used have direct analogues in 3D.
\end{abstract}

Key words. Inverse scattering, Helmholtz equation, geometric optics, microlocal analysis.

AMS subject classifications. 34L25, 35J05, 78A05.

\section{Introduction}

We consider an application in which an autonomous observer, sent into an unknown environment, must discover the locations of signal sources in an efficient manner. The unknown environment contains non-penetrable solid obstacles, $\Omega$, that should be avoided along the observer's path. We assume that $\Omega$ is bounded and has piecewise smooth boundaries and that the signal strength $u$ satisfies the Helmholtz equation

$$
\Delta u(x)+k^{2} \eta(x)^{2} u(x)=\sum_{n=1}^{N} \delta\left(x-x_{n}\right) \text { for } x \in \mathbb{R}^{2} \backslash \Omega,
$$

with suitable reflection boundary conditions on $\partial \Omega$ and radiation boundary conditions as $|x| \rightarrow \infty$. The coefficient $\eta=\eta(x)$ is the index of refraction and $\lambda$ and $k=2 \pi / \lambda$ are the wavelength and wavenumber respectively. The signal sources are modeled by Dirac- $\delta$ distributions on the right hand side of (1.1). The observer must determine the number $N$ of sources and their locations $x_{n}$ based on sparse measurements of the signal in the environment.

The task of source discovery is an inverse problem that uses the given wave field values on some subset of the domain to recover the source locations. In order to determine the locations of the sources, some mathematical models about the sourcesensor relationship is presumed. A set of parameters that characterize the sources is inferred from the models and these parameters need to be determined from the data that is typically collected from dense arrays of sensors at fixed locations in a

*Received: July 27, 2010; accepted (in revised version): January 28, 2011. Communicated by Lenya Ryzhik.

${ }^{\dagger}$ Department of Mathematics, University of California, Los Angeles, CA 90095 (ylanda@math. ucla.edu). Landa's research is supported by NSF DMS-0914561 grant and an ARO MURI grant.

$\ddagger$ Department of Mathematics, University of Texas at Austin, TX 78704 (nicktan@math.utexas. edu). Tanushev's research is supported by NSF grants No. DMS-0914465, DMS-0914840, and DMS0636586 (UT Austin RTG), and ARO MURI U. South Carolina Subaward 07-1409.

$\S$ Department of Mathematics, University of Texas at Austin, TX 78704, (ytsai@math.utexas.edu). Tsai's research is supported by NSF grants No. DMS-0914465, DMS-0914840 and ARO MURI U. South Carolina Subaward 07-1409. 
simple domain. The data is usually given in the form of measurements of the wave field on some subset of the domain. A standard way of solving this inverse problem for finding the source function is to minimize the $L^{2}$ norm of the difference between the collected data and simulated data computed with a candidate source function. This minimization is typically achieved through an iterative procedure that optimizes the parameters of the candidate source function. To make the inversion more stable, this least square approach may be regularized with additional terms. Thus, a numerical solution of the forward problem, in our case Equation (1.1), is a necessary step for solution methods based on such an approach. When the wavelength $\lambda$ is small compared to overall size of the computational domain, the oscillatory behavior of the solution makes direct numerical simulation of (1.1) computationally expensive: a substantial number of grid points per wavelength is required to maintain constant accuracy. For sufficiently high frequencies, it is unrealistic to compute the wave field directly. For example, visible light has wavelengths of the order of nanometers. Thus, direct numerical simulations over dimensions on the order of meters is nearly impossible. Audible sound frequencies are in the $20 \mathrm{~Hz}$ to $20 \mathrm{kHz}$ range, which corresponds to wavelengths of approximately $15 \mathrm{~m}$ to $15 \mathrm{~mm}$. For problems such as underwater acoustics, even when high frequency techniques become applicable at large distances, simulations of the wave field in a step of an iterative method is unfeasible.

Inverse problems for wave phenomena typically involve some form of backpropagation of the waves, or time reversal, from the sensors back into the domain; this is called imaging or migration in geophysics [32]. Different approximations of the waves have been explored for the back-propagation; see e.g. the MUSIC algorithm [45] and Kirchoff migration [32]. The goal of the time reversal problem [4, 20,3, 19] is similar to the source discovery problem considered in this paper, however, the underlying physical and mathematical structure is very different. In time reversal, one tries to locate the source by reversing the wave field while estimating the effective Green's function without knowing details of the random fluctuation present in the medium. A key mechanism in time reversal imaging is averaging, which can be realized by employing multiple uncorrelated sensors and frequencies [27]. Common assumptions are that the wavelength of the signal is much larger than the scale of the random fluctuations, that there is scale separation of the medium and that statistical properties of the fluctuations are known. Our approach assumes signals with wavelength smaller than the scatterers and a smoothly varying medium.

A rigorous study of inverse scattering for the high frequency Helmholtz equation in the framework of compressive sensing is performed by Fannjiang in $[25,26]$. In this setting, inhomogeneities of the medium are represented by a finite number of point scatterers on a square lattice. The reconstruction of the scatterers from the scattering amplitude succeeds only probabilistically due to intrinsic limitations of the problem formulation in the framework of compressive sensing. The number of measurements is proportional to the sparsity of the scatterers and a factor of $\log (m)$, where $m$ is the size of the square lattice. In our proposed algorithm, the upper bound on the number of measurements required to detect the sources in a planar environment with unknown convex scatterers is $3 N+3 O$, where $N$ and $O$ are the number of scatterers and sources respectively.

When the obstacles are unknown, the environment needs to be mapped out as the observer moves. The previous work of $[37,35,38]$ on reconstructing the obstacles in unknown domains is convenient in this regard. A piecewise high-order approximation of the scattering surfaces can be obtained on-the-fly by processing the visibility point 
cloud data collected along the observer's path. Additionally, the visibility information is necessary to avoid collisions with the obstacles. In [6] and [36], the authors extend the domain mapping algorithms of [37] and [35] for point source discovery in complicated environments with obstacles using an approach based on reciprocity of the linear partial differential operators as well as the maximum principle for the associated elliptic problems. This approach fails in the case of the Helmholtz equation which does not satisfy a maximum principle due to the oscillatory structure of the solution.

Source identification problems have been considered in many other settings as well. In $[39,34]$, the authors propose to recover the exact locations of multiple sources in a Poisson equation, given the initial guess for the locations and Dirichlet data on the boundary of the domain. To accomplish this, they use the special form of the free space Green's function for Poisson's equation. Other related topics can be found in [31, 22, 23, 15, 40]. Convex optimization techniques are employed in [14] to identify multiple sources in Poisson equation in simple domains without obstacles. In contrast to the typical inverse problem settings, we consider complicated domains, the possibility of placing the sensors freely in the domain, and the necessary coupling with visibility information in the case of unknown environments.

In this paper, we consider the high frequency regime in which the wavelength $\lambda$ is much smaller than the size of the scatterers in the domain. In this regime, the signal travels along straight lines in free space and reflects from scattering surfaces following Snell's law (angle of incidence equals angle of reflection). We reduce the complexity and cost of the numerical computations by representing the solution with a fixed number of unknowns which are independent of the frequency locally near measurement locations. The quantities that describe the wave field can be obtained via a geometric optics (GO) interpretation of the solution, which relies on asymptotic expansions $[33,24,5,2]$. One natural idea from these methods is to represent the solution of (1.1) in terms of an explicit dependence on frequency and functions independent of frequency. In contrast to direct methods, the accuracy of asymptotic methods improves as frequency increases.

In this high frequency setting, we propose a source discovery algorithm for a domain in which the scatterers are not known a priori but can be mapped via the visibility at the locations where wave data is gathered. Furthermore, we do not assume the knowledge of the total number of sources present in the domain. There are two key components in our algorithm. The first is to identify all significant local wave directions passing through the observation point and the second is to trace these rays back to the sources and form an image of the possible source locations using the fact that the sources must lie at the intersection of rays from multiple observation points. The problem of identifying the significant local wave directions was formulated by Benamou et al. in [2]. We use their framework with one key difference: Since we are looking for a small number of directions in a possibly noisy signal, we use an $L^{1}$ constrained minimization instead of the Tikhonov regularized $L^{2}$ minimization proposed in [2] to solve the frequency-independent sparse linear system. We employ a fast Bregman iterative solver $[18,47,7]$ to yield an accurate solution, which is robust against the noise in the initially sampled signal. This solution contains information about the number of waves crossing at the observer's location as well as their directions. It is worth pointing out that the minimization problem is solved in a lower dimensional domain with a cost that is independent of the wave number. The second key component involves a source imaging procedure which is based on 
back-propagation of the detected incoming wave. The visibility algorithm that we employ for unknown environments [37, 35], summarized in Algorithm 2, provides the necessary information for determining the validity of the back-propagated rays and for computing the ray reflections. One can also use these secondary rays to locate the sources, however, our proposed algorithm does not use these reflected rays, as tracing reflections off convex surface is inherently sensitive to errors in the ray directions and surface geometry. Nonetheless, we point out to the reader that these rays can be used to guide the selection of new measurement locations, and this is in accordance with our formulation of the source location problem.

Our strategy for source discovery has a natural limit due to the nature of the scattering problem. When the number of connected scatterers between the measurement locations and the sources is too big, the wave field at the measurement location may be highly attenuated and may contain rays coming from every direction. Additionally, when the size and the number of scattering solids in the domains scales in a certain special way with the wavelength, the Helmholtz formulation will no longer be adequate. In these cases, one has to resort to a different formulation using effective equations [30]. This is one of our current research directions. However, we shall demonstrate that our high frequency wave based algorithm does provide good solutions for source identification in a domain containing many convex scatters whose size is only on the order of 10 wavelengths.

The rest of the paper is outlined as follows. In Section 2, we describe how to estimate wave directions at an observation location. In Subsection 2.1, we present the mathematical formulation of the inverse problem defined in [2]. We detail the numerical solution of the inverse problem and introduce the results based on constrained $L^{1}$ minimization problem in Subsection 2.2. Our proposed strategy has two major components: imaging the sources based on observations from a set of fixed locations and adaptively choosing the observation locations, so that the sources can be identified in finitely many observations. Section 3 contains the description of the functional used to image the sources in the domain. The adaptive algorithm for choosing the observing locations, along with examples, is described in Section 4.

\section{Determining wave directions at an observation location}

As mentioned in the introduction, a major component of our algorithm is estimating the wave directions at an observation location. To accomplish this, we use a modified version of the algorithm presented in [2]. We begin by describing in detail the formulation of [2] and our $L^{1}$ modification of the minimization. We also present comparisons between our numerical solution method and the Tikhonov regularized $L^{2}$ formulation presented in [2].

2.1. Mathematical formulation. The theory of geometric optics allows for the approximation of a high frequency wave field by a phase and amplitude computed on a special set of curves through space. The curves, phase, and amplitudes are independent of the frequency and hence provide a frequency independent method for numerical simulations. In a medium with constant wave speed, the curves (or rays) are straight lines and the amplitudes can be determined by the geometric spreading of the rays.

The asymptotic representation of the solution of (1.1) is usually based upon superpositions of the geometric optics ansatz [1, 24]

$$
u(x) \simeq \sum_{n=1}^{M} A_{n}(x) e^{i k \phi_{n}(x)} .
$$


Each phase $\phi_{n}(x)$ and amplitude $A_{n}(x)$ are independent of frequency (away from caustics) and they are real valued solutions of the Eikonal and transport equations:

$$
\begin{aligned}
\left|\nabla \phi_{n}\right| & =\eta(x) \\
2 \nabla \phi_{n} \cdot \nabla A_{n}(x)+A_{n} \triangle \phi_{n} & =0 .
\end{aligned}
$$

The rays are the projection of the bicharacteristics of the Eikonal equation onto $x$ space.

If the amplitudes $A_{n}$ and phases $\phi_{n}$ are smooth in a neighborhood of an observation point $y$, a first order linear approximation around $y$, along with (2.1), gives a local plane wave decomposition

$$
u(x) \simeq \sum_{n=1}^{M} B_{n}(y) e^{i k(x-y) \cdot \nabla \phi_{n}(y)},
$$

where $B_{n}(y):=A_{n}(y) e^{i k \phi_{n}(y)}$ is the "complex amplitude".

In [2], the authors pose the following inverse problem:

Given an analytical or numerical solution $u(x)$ in a neighborhood of a

fixed observation point $y$, determine the number $M$ of waves crossing

at $y$ and compute the $G O$ quantities $\left(B_{n}, \nabla \phi_{n}\right)$ for $n=1, \ldots M$.

By solving this problem, we are able to approximate the solution by smooth functions $A_{n}(x)$ and $\phi_{n}(x)$, which can be represented by a fixed number of unknowns, independent of the frequency. Note that for large $k$, the function $B_{n}(y)$ is highly oscillatory, and it may be difficult to recover $\phi_{n}(y)$ from a numerical approximation. Its modulus, on the other hand, is smooth and equal to $\left|A_{n}(y)\right|$.

The numerical algorithm proposed in [2] samples the wave field locally along the circumference of a small circle centered at the observation point $y$. By analyzing this data, information about the number of waves crossing at $y$ and their corresponding complex amplitudes can be extracted. Below we describe the numerical formulation of the problem as well the filtering procedure based on the Jacobi-Anger formula, introduced in [2].

We assume the solution $u$ of the Helmholtz Equation (1.1) can be sampled on a circle $C_{r}(y)$ of radius $r:=\alpha / k \eta(y)$ around the observation point $y$ :

$$
U_{\alpha}(s):=u\left(C_{r}(y)\right)
$$

Here, $\alpha$ is a given positive number and $s$ parameterizes the circle

$$
C_{r}(y)=y+r(\cos \theta(s), \sin \theta(s)) .
$$

Let $d_{n}(y)=\left(\cos \theta_{n}, \sin \theta_{n}\right)$ denote the direction of propagation of the $n^{\text {th }}$ wave,

$$
\nabla \phi_{n}(y)=\eta(y) d_{n}(y) \text {. }
$$

Along the circle $C_{r}(y)$, the restriction of the plane wave superposition (2.4) has the form

$$
U_{\alpha}(s) \simeq \sum_{n=1}^{M} B_{n}(y) e^{i \alpha(\cos \theta(s), \sin \theta(s)) \cdot d_{n}(y)} .
$$

The goal is to recover the ray directions from the knowledge of $U_{\alpha}(s)$. The idea of [2] is to remove the effect of the exponential factor by filtering and to calculate a function 
$\beta_{\alpha}(s)$ that has distinct peaks along the wave vectors and approaches $B_{n}$ in the limit as $\alpha$ approaches infinity. To accomplish this, we use the 2D Jacobi-Anger expansion [17]:

$$
e^{i \alpha(\cos \theta(s), \sin \theta(s)) \cdot d_{n}(y)}=e^{i \alpha \cos \left(\theta_{n}-\theta(s)\right)}=\sum_{l=-\infty}^{\infty} i^{l} J_{l}(\alpha) e^{-i l\left(\theta_{n}-\theta(s)\right)},
$$

where $J_{l}(\alpha)$ is the Bessel function of order $l$. Inserting (2.9) into (2.8), we get the expansion

$$
U_{\alpha} \simeq \sum_{l=-\infty}^{\infty} i^{l} J_{l}(\alpha)\left(\sum_{n=1}^{M} B_{n} e^{i l\left(\theta(s)-\theta_{n}\right)}\right) .
$$

Note that when $\alpha$ is held fixed, $J_{l}(\alpha)$ goes to zero more than exponentially fast as $|l| \rightarrow \infty$ [17]. Therefore, the series can be truncated at some threshold $|l| \leq L(\alpha)$ [16]. A suitable choice of $L(\alpha)$ in our computations is provided in $[2,16]$ :

$$
L(\alpha)=\alpha+8 \log (\alpha) .
$$

We now introduce the space of square summable sequences

$$
l^{2}=\left\{\gamma_{l}=\frac{1}{2 \pi} \int_{S^{1}} \alpha(s) e^{-i l \theta(s)} d s,-\infty<l<\infty, \sum_{l=-\infty}^{\infty}\left|\gamma_{l}\right|^{2}<\infty\right\},
$$

and let the Fourier transform on the unit circle $\mathcal{F}: L^{2}\left(S^{1}\right) \mapsto l^{2}$ be given by

$$
(\mathcal{F} A(s))_{l}:=\frac{1}{2 \pi} \int_{S^{1}} A(s) e^{-i l \theta(s)} d \sigma(s),
$$

with inverse

$$
\left(\mathcal{F}^{-1} \gamma\right)(s)=\frac{1}{2 \pi} \sum_{l} \gamma_{l} e^{i l \theta(s)}
$$

Let $D_{\alpha}: l^{2} \mapsto l^{2}$ be the division and restriction operator

$$
\left(D_{\alpha} \gamma\right)_{l}:= \begin{cases}\frac{2 \pi}{2 L(\alpha)+1} \frac{1}{i^{l} J_{l}(\alpha)} \gamma_{l}, & |l| \leq L(\alpha), \\ 0, & \text { otherwise }\end{cases}
$$

assuming $J_{l}(\alpha) \neq 0$ for $|l| \leq L(\alpha)$. We now define the function

$$
\beta_{\alpha}:=\mathcal{F}^{-1} D_{\alpha} \mathcal{F} U_{\alpha} .
$$

As a result of the above spectral inversion procedure, the function $\beta_{\alpha}$, computed from the local values around the observation point, will have sharp peaks in the directions of propagation of the waves passing through $y$ when $\alpha$ is large enough [2]. In other words, for a fixed $s$ we have

$$
\lim _{\alpha \rightarrow \infty} \beta_{\alpha}(s)= \begin{cases}B_{n}, s=d_{n} \\ 0, & \text { otherwise. }\end{cases}
$$

Thus, the larger the parameter $\alpha$, and therefore the size of the observation circle, the better the accuracy of the result. On the other hand, the size of the neighborhood must not exceed the domain of validity of the local plane wave approximation (2.4). In the following section we will discuss the numerical approximation of $\beta_{\alpha}$. 
2.2. Numerical solution. The key to finding the unknown number of rays, their complex amplitudes and directions is the function $\beta_{\alpha}$ defined in (2.16). Therefore, we first compute a numerical approximation of $\beta_{\alpha}$ on a uniform discretization of the unit circle.

We begin by introducing a uniform grid $\left\{\tilde{\theta}_{m}\right\}$ with $M \geq 2 L(\alpha)+1$ points on the unit circle,

$$
\tilde{\theta}_{m}=m \Delta \theta, \quad \Delta \theta=\frac{2 \pi}{M}, \quad m=0, \ldots, M-1 .
$$

Let $\left\{\tilde{U}_{m}\right\}$ be the grid function that samples the given function $U_{\alpha}(s)$ on the grid along the observation circle,

$$
\left\{\tilde{U}_{m}\right\}=U_{\alpha}\left(\tilde{d}_{m}\right), \quad \tilde{d}_{m}=\left(\cos \tilde{\theta}_{m}, \sin \tilde{\theta}_{m}\right) .
$$

Then, the discretized version of $(2.16), \beta_{\alpha}\left(\tilde{\theta}_{m}\right) \simeq b_{m}$, is given by

$$
\left\{b_{m}\right\}=2 \pi \mathrm{FFT}^{-1}\left\{\frac{\left(\mathrm{FFT}\left\{\tilde{U}_{m}\right\}\right)_{l}}{M i^{l} J_{l}(\alpha)}\right\},
$$

where we have taken precisely

$$
M=2 L(\alpha)+1,
$$

since FFT maps $N$ dimensional vectors to $N$ dimensional vectors.

We remark that the same discretization can applied to the original plane wave representation (2.8) to obtain the linear system

$$
\tilde{U}_{n}=\sum_{m=1}^{M} \tilde{b}_{m} e^{i \alpha \tilde{d}_{n} \cdot \tilde{d}_{m}}, \quad n=0, \ldots, M-1 .
$$

The resulting circulant matrix could be solved for $\left\{\tilde{b}_{m}\right\}$ directly at a cost comparable to solving (2.18), $O(M \log M)$ [29]. However, as $M$ grows, the condition number of the matrix deteriorates rapidly and the problem becomes very ill-conditioned when $M \geq L(\alpha)[2]$. The FFT-based Jacobi-Anger inversion proposed in [2] is, in fact, a stabilized approximation $\left(\tilde{b}_{m} \sim b_{m}\right)$ of the standard fast way to solve this circulant matrix problem.

Let us rewrite (2.18) as a linear system with $b=\left\{b_{m}\right\}$ as the unknown:

$$
\begin{array}{r}
G b=\hat{U}, \quad G=\left\{g_{l, m}\right\}, \quad g_{l, m}=M i^{l} J_{l}(\alpha) e^{-i l \tilde{\theta}_{m}}, \quad \hat{U}=\left\{\hat{U}_{l}\right\}, \\
l=-L(\alpha), \ldots, L(\alpha), \quad m=0, \ldots, M-1 .
\end{array}
$$

The matrix $G$ is $(2 L(\alpha)+1) \times M$ and, since in this formulation we are not using the inverse FFT, the number of samples along the unit circle is not restricted by the constraint (2.19) on $M$. Thus, in general, the system (2.21) may be overdetermined. However, in our computations, we avoid the extra parameters by considering the square version of (2.21) under the constraint (2.19). It is clear that zeros of the Bessel function $J_{l}(\alpha)$ may be a problem in the numerical solution of this system. Even 
though some of the small values of the Bessel function are eliminated by truncating the series when $|l| \rightarrow \infty$, the Bessel function as a function of $\alpha$ may have zeros, and there is no guarantee that $\alpha$ is not close to one of them. The rows at which $J_{l}(\alpha)=0$ make the effective row space smaller. This can be resolved by introducing suitable regularization. The numerical algorithm proposed in [2] consists of two steps involving Tikhonov regularization procedure followed by simple filtering and a non-linear optimization procedure. The Tikhonov regularized $L^{2}$ system is

$$
\left(G^{*} G+\epsilon I\right) b_{\epsilon}=G^{*} \hat{U},
$$

where $I$ is the identity matrix and $\epsilon$ is the regularizaiton parameter. The exact Tikhonov regularization would consist of choosing $\epsilon$ so that the relative error between the actual and regularized problem is smaller than a prescribed precision. The inversion formula (2.18) becomes

$$
\left\{b_{m}^{\epsilon}\right\}=2 \pi \mathrm{FFT}^{-1}\left\{\frac{\hat{U}_{l} M J_{l}(\alpha)}{i^{l}\left[M^{2} J_{l}(\alpha)^{2}+4 \epsilon \pi^{2}\right]}\right\}
$$

and remains bounded even when $J_{l}(\alpha)$ is zero or close to zero. In the post-processing step, the data obtained from the above spectral inversion is used as initial data in the fitting of the plane wave expansion (2.4) to the values sampled along the observation circle. This is done by non-linear minimization of the residual. As a result, the accuracy of the rays' complex amplitudes and directions can be significantly improved.

The above approach of [2] does not take into account the sparsity of the solution, nor does is seem to be robust to noise in the signal. Thus, we propose to modify this approach using a convex optimization technique, namely, constrained $L^{1}$ minimization. Our modified approach exploits the sparse nature of the solution, is computationally efficient, and is robust to signal noise.

2.2.1. Fast $L^{1}$ solution methods. The Tikhonov regularization of the system described in [2] typically returns solutions with all non-zero entries. Instead, we are interested in a solution with the least number of non-zeros which would correspond to the directions of the rays. Based on the linear system (2.21) we formulate an $L^{1}$ constrained minimization problem, which resembles the formulation of compressive sensing (CS) $[21,9,10]$. The theory of compressive sensing determines how well a sparse signal can be reconstructed from measurements in a different basis. The key results reduce sparsity to an equivalent convex minimization, specifically, $L^{1}$ minimization, which is known to be of great importance in problems of this type.

A CS problem may be described as follows. Suppose we observe $f=A u$, where $u \in$ $\mathbb{R}^{n}$ is the sparse function we wish to reconstruct, $f \in \mathbb{R}^{m}$ are available measurements, and $A$ is a known $m \times n$ matrix. In typical compressive sensing settings, we are interested in the underdetermined case with fewer equations than unknowns, i.e. $m<n$, and wish to reconstruct $u$ with good accuracy.

The most straightforward way to solve the CS problem is to consider the minimization problem

$$
\min _{u \in \mathbb{R}^{n}}\left\{|u|_{0}: A u=f\right\}
$$

which can be solved approximately by matching pursuit [41, 44, 13]. However, the matching pursuit method is not computationally efficient for typical applications and it does not guarantee the finding of a global minimizer of (2.24) in general. 
It has been shown in $[9,10,21]$ that the solution to an equivalent convex optimization problem, specifically $L^{1}$ constrained minimization,

$$
\min _{u \in \mathbb{R}^{n}}\left\{|u|_{1}: A u=f\right\},
$$

recovers $u$ exactly, provided the linear measurements satisfy the Restricted Isometry Property (RIP) hypotheses of $[12,11,8]$.

There has been a recent burst of research in compressive sensing, which involves solving (2.25). This was led by Candès et al. [11, 12, 9], Donoho [21], and others [47]. Conventional linear programming techniques are not tailored for the large scale dense matrices and sparse solutions that arise in (2.25). A robust and efficient way to solving $L^{1}$ related regularized problems was developed by Osher et al. [18, 28, 47, 7, 43]. These kinds of algorithms are generally referred to as Bregman iterative methods. Bregman iterative techniques consider minimizing a problem of the form

$$
\min _{u \in \mathcal{H}} J(u)+H(u, f),
$$

where $J$ and $H$ are two convex functions defined on a Hilbert space $\mathcal{H}$. The Bregman iteration algorithm proposed in [47] is written in two lines:

$$
\begin{aligned}
& u^{k+1}=\arg \min _{u \in \mathbb{R}^{n}}\left\{J(u)-J\left(u^{k}\right)-\left\langle u-u^{k}, f^{k}\right\rangle+H(u, f)\right\}, \\
& f^{k+1}=f^{k}-\nabla_{u} H\left(u^{k+1}, f\right),
\end{aligned}
$$

with $u^{0}=0$ and $f^{0}=0$. This algorithm generates a sequence $\left\{u^{k}\right\}$ such that $H\left(u^{k}, f\right)$ decreases monotonically. Furthermore, it has been proven in [47] that if $J(u) \in C^{2}(\Omega)$ and is strictly convex in $\Omega$, then $H\left(u^{k}, f\right)$ decays exponentially whenever $u^{k} \in \Omega$ for all $k$.

The compressive sensing type of problems, such as (2.25), corresponds to the special case where $J(u)=|u|_{1}$ and $H(u, f)=\frac{1}{2}|| A u-\left.f\right|_{2} ^{2}$. The minimizer of $J(u)$ is sought under the constraint $H(u, f)=0$. In this case, the sequence $u^{k}$, obtained through Bregman iterations (2.27), converges in finitely many steps to the solution of (2.25). Furthermore, for such quadratic fitting term type problems, the Bregman iteration (2.27), after some manipulation, can be written as

$$
\begin{aligned}
u^{k+1} & =\arg \min _{u \in \mathbb{R}^{n}}\left\{J(u)+\frac{1}{2}\left\|A u^{k}-f^{k}\right\|^{2}\right\}, \\
f^{k+1} & =f^{k}+f-A u^{k+1},
\end{aligned}
$$

with $f^{0}=0$ and $u^{0}=0[47,42]$. Thus, the Bregman iteration scheme solves the constrained minimization problem via solutions of a sequence of unconstrained problems.

To further improve on the computational simplicity, the linearized Bregman algorithm was devised in [18]. By linearizing the quadratic fitting term around the previous iterate $u^{k}$ and seeking the minimizer near it, the two-line linearized Bregman algorithm can be written component-wise as

$$
\begin{aligned}
u^{k} & =\operatorname{shrink}\left(v^{k-1}, \mu\right):=\operatorname{signum}\left(v^{k-1}\right) \max \left\{\left|v^{k-1}\right|-\mu, 0\right\}, \\
v^{k} & =v^{k-1}=\delta A^{\top}\left(A u^{k}-f\right),
\end{aligned}
$$

for $\mu, \delta>0$. This algorithm takes advantage of the simple exact solution formula for the scalar problem:

$$
\arg \min \left(\mu|u|+\frac{1}{2}|f-u|^{2}\right)=\operatorname{shrink}(f, \mu) .
$$


The linearized Bregman algorithm is proven to solve the original $L^{1}$ constrained minimization problem for sufficiently large $\mu[46]$.

Since Bregman iterative algorithms demonstrate great speed, accuracy, and potential for extensions [43], we use a Bregman iterative method for detecting the ray directions. We begin by transforming the complex-valued matrix $G \in \mathbb{C}^{M \times M}$ and the measurements vector $\hat{U} \in \mathbb{C}^{M}$ from (2.21) to real-valued matrix $A$ and vector $f$ by separating real and imaginary parts:

$$
A \in \mathbb{R}^{2 M \times 2 M}, f \in \mathbb{R}^{2 M}: A=\left(\begin{array}{cc}
\Re G & \Im G \\
-\Im G & \Re G
\end{array}\right), f=\left(\begin{array}{c}
\Re \hat{U} \\
\Im \hat{U}
\end{array}\right) .
$$

However, the solution to our problem can be either non-unique with only few measurements or non-existent when the measurements are not consistent due to noise or other uncertainties. Thus, we replace the exact equality in (2.25) by an inequality with an $L^{2}$ norm:

$$
\min _{u \in \mathbb{R}^{n}}\left\{|u|_{1}: \| A u-f||<\epsilon\right\},
$$

where $\epsilon>0$ is a small number. We now apply the linearized Bregman iterations (2.29) to solve the $L^{1}$ constrained minimization problem (2.32) to obtain the solution $u \in \mathbb{R}^{2 M}$, which can then be easily transformed back into complex form $b_{m} \in \mathbb{C}^{M}$. The absolute value of $b_{m}$ has peaks in the directions of the rays and corresponds to the complex amplitude $B_{n}$ in (2.4).

2.3. Summary of Algorithm for determining wave directions. The steps necessary to determine the wave directions at each observation location can be summarized as follows:

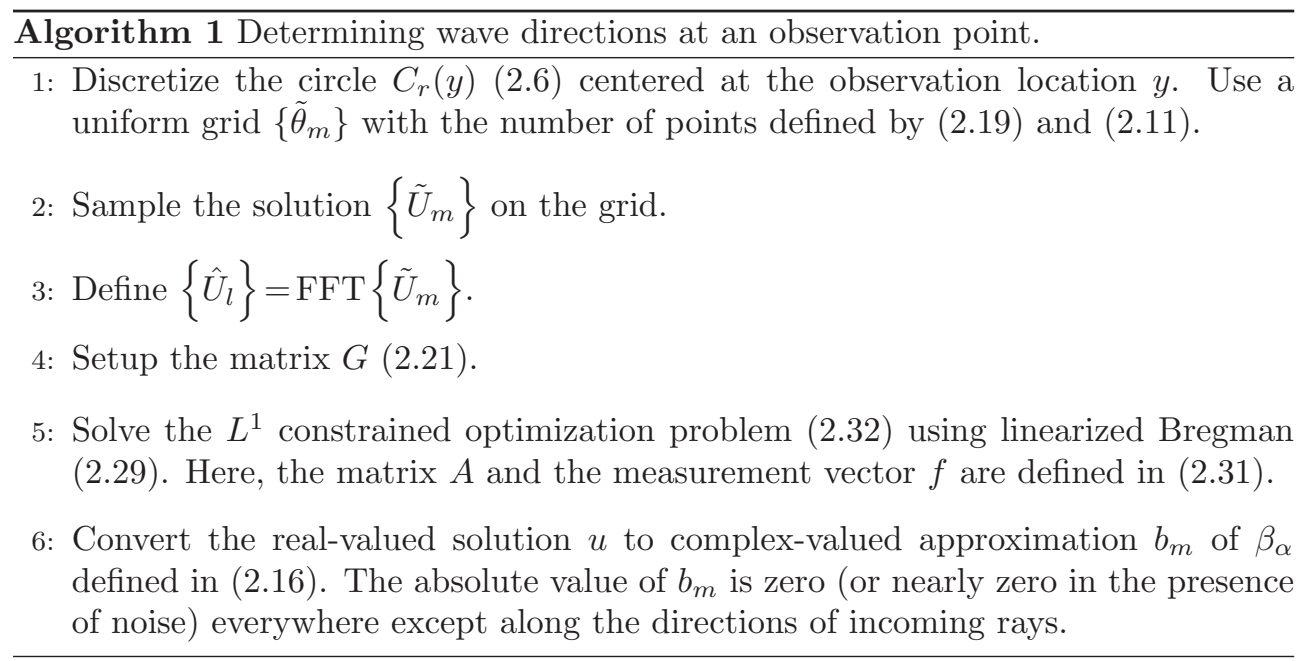

2.4. Results. The following simple model problem in free space is used in the experiments described in this section:

$$
\Delta u_{k}+k^{2} u_{k}=\sum_{n=1}^{N} 4 i \sqrt{k} \delta_{x_{n}},
$$


where $N=5$. The Dirac- $\delta$ masses are centered at the source locations $\left\{x_{n}\right\}_{n=1}^{N}=$ $\{( \pm 0.8,-0.6),( \pm 0.4,-0.6),(0,-0.6)\}$ and normalized so that the exact solution can be written as the sum of Hankel functions with decaying amplitudes centered at $x_{n}$ :

$$
u_{k}(x)=\sum_{n=1}^{N} \sqrt{k} H_{0}^{1}\left(k\left|x-x_{n}\right|\right) .
$$

We would like to recover the ray directions with nonzero amplitudes in the local approximation of the wave field at the observation point $y=(0,0.2)$. The exact solution, observation point along with the observation circle, and the point sources are shown in Figure 2.1 (a). The performance in the presence of noise of the constrained $L^{1}$ minimization is compared to the Tikhonov regularized $L^{2}$ minimization in Figure 2.1 (b-c). In this experiment, the wave number is $k=10^{3}$. The radius of the observation circle is $r=\alpha / k$, where $\alpha$ is a variable parameter set to $3 \pi$. The number of measurements along the observation circle is $M=55$.

Figure 2.1 (b) depicts the solution of the Tikhonov regularized $L^{2}$ system (2.22) using the inversion formula (2.23). Note, however, that we do not apply the postprocessing step discussed in [2]. The vertical dashed lines indicate the exact angular positions of the point sources with respect to the observer. The exact solution would thus have peaks along the dashed lines and be zero everywhere else. The $L^{2}$ solution is almost never zero and has many oscillations away from source locations. As the Signal-to-Noise Ratio (SNR) decreases, the solution becomes more oscillatory, so that the true peaks are lost in the spurious oscillations.

In contrast, the solution of the $L^{1}$ constrained minimization problem (2.32), shown in Figure 2.1 (c), is zero almost everywhere and does not deteriorate much in the presence of noise. The spurious oscillations that appear as SNR decreases have small amplitudes and can be easily filtered out by simple thresholding.

In Figure 2.2, the maximum error in the ray directions $\theta_{n}$ is plotted as a function of $\alpha$ (proportional to the radius of the observation circle). The number of measurements along the circle is calculated using (2.19) and (2.11). The error in the ray directions is bounded below by the grid resolution. When $\alpha$ is very small, the radius of the observation circle is small, and the mesh is rather coarse, consisting of just a few points. The error decreases as $\alpha$ becomes larger. However, when $\alpha$ is too big, the error increases again possibly due to the violation of the local plane wave approximation (2.4).

In the absence of noise, the accuracy of the solution of the $L^{1}$ problem is comparable to the accuracy of the results obtained solving Tikhonov regularization problem. Further convergence analysis is provided in [2].

\section{Source discovery via back-propagating rays}

In this section, we describe how to determine the source locations from a fixed set of observation points without complete knowledge of the scattering objects in the domain. At each observation location $O$, we assume that a set of incoming wave directions are detected by the algorithm described in the previous section. Associated with each of these directions is a ray coming either directly from a source or indirectly via reflections. Thus, all the sources must lay at intersections of these rays, when no error is present in the detection of the incoming wave directions or in the reflection angles. A natural idea is to propagate rays back into the part of the domain that has been explored by the navigation algorithm and look for the sources among the intersections of these rays. Instead of looking for these intersections one by one, which 


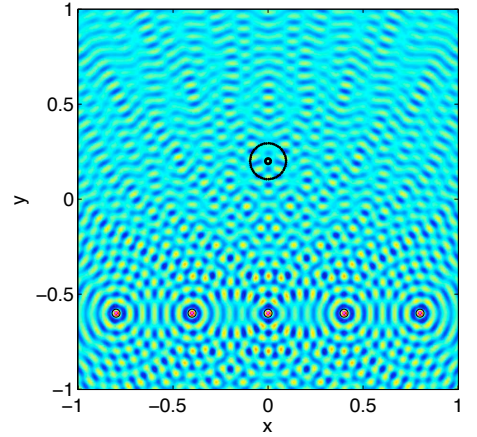

(a) Problem setup.

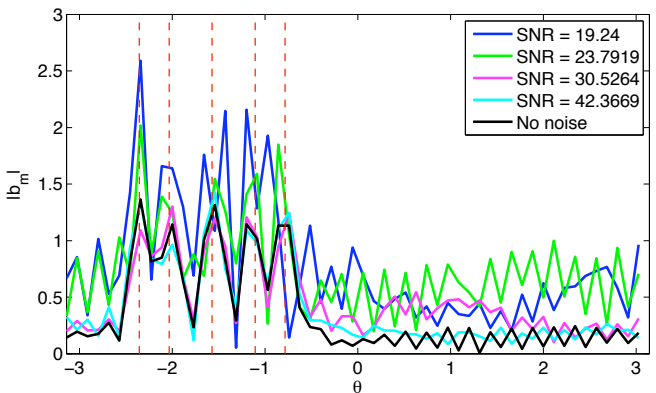

(b) Solution of the Tikhonov regularized $L^{2}$ system (2.22) using the inversion formula (2.23).

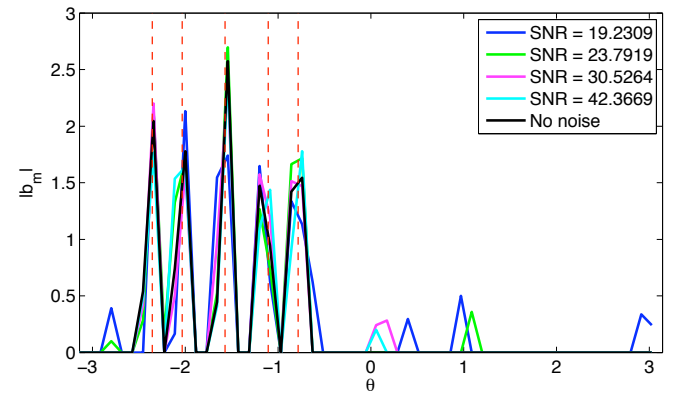

(c) Solution of the $L^{1}$ constrained minimization problem (2.32) using linearized Bregman iterations.

FIG. 2.1. Solution of the Helmholtz equation with five sources at different noise levels. Vertical dashed lines in subfigures (b) and (c) indicate the exact angular positions of the sources with respect to the observer's location.

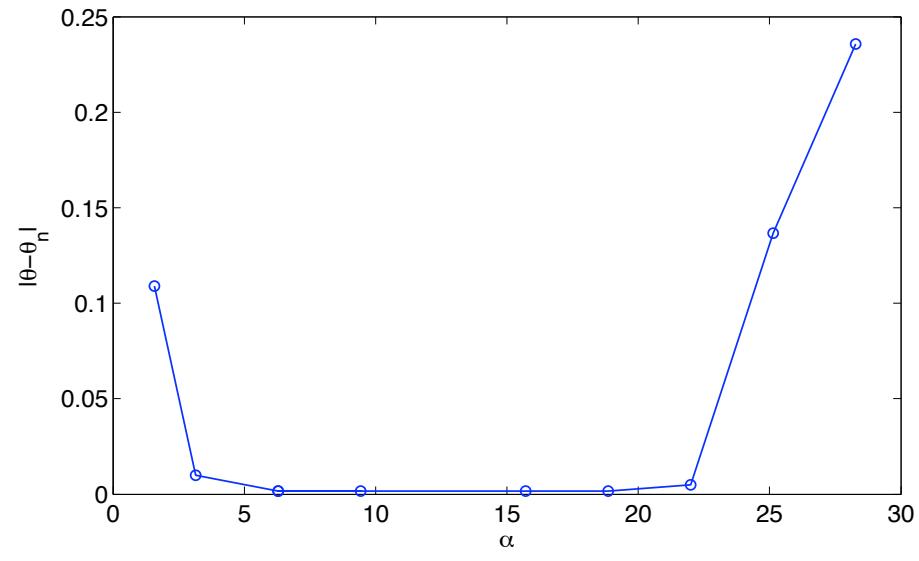

FIG. 2.2. The error in the ray directions $\theta_{n}$ as a function of $\alpha$. Fixed wave number $k=10^{3}$. The radius of the observation circle is $r=\alpha / k$ and the number of measurements along the circle is given by (2.19). 
has many drawbacks such as instability, we propose to image the sources by forming a density that reflects the estimated positions of the sources. After a renormalization, the image produced by our algorithm can be thought of as a probability density.

At each observation location $O$, we propagate rays back towards the detected wave directions, which we will refer to as the primary rays. The visibility function at $O$ provides all of the necessary information to find the reflections of any primary rays that reach an obstacle, since the obstacle geometry is resolved by the visibility function and we can find the normal to the obstacle boundary locally, which determines the angle of reflection through Snell's law. However, after a ray reflects, its secondary part can enter regions of the domain which have not been explored yet, thus, there may not be the necessary information to find how they reflect from obstacles. Furthermore, the errors in ray direction and in the normal vector to the obstacle boundaries tend to significantly degrade the information contained in the secondary rays. Thus, we have found that using only the primary rays is more efficient and provides a better image compared to using also the secondary reflected parts of the rays. In addition, the solution for the Helmholtz equation decays rapidly away from the source, supporting the idea that most of the wave direction information at each observation location is contained in the primary rays. For the remainder of this section, we will refer to the primary rays as simply the rays.

We remark that given a fixed set of observation locations and the wave directions at each of them, there are many different ways of forming an imaging functional. In designing such a functional, we have found that certain properties are necessary to produce a good image. We will present our arguments about why these properties are important and then summarize them in a list.

3.1. Imaging functional. First, we begin by looking at the simple case of only one observation location. At such a location $O$, we back-propagate each detected incoming wave direction. Each ray begins at $O$ and terminates by either reaching an obstacle or by exiting the domain, which can be determined by the visibility information at $O$. Thus at the observation location $O$, we obtain a set of backpropagated line segments $\left\{R_{m}(O): m=1,2, \cdots, M_{O}\right\}$. A source may reside anywhere along these line segments. Of course, due to reflections and other errors, this is not necessarily the case. However, given no other information, our best analysis of the situation is that a source is most likely to be somewhere on a ray. Thus, we form a distribution that decays exponentially away from each line segment. Furthermore, due to the angular discretization of the directions, we decrease the decay rate based on the distance away from the observation location:

$$
I^{S}(x ; O)=\max _{m \in[1, M]}\left[\exp \left(-\frac{\nu_{1}}{\left|O-x_{m}^{*}\right|^{2}}\left|x-x_{m}^{*}\right|^{2}\right)\right],
$$

where $O$ is the observation location, $\nu_{1}$ is a positive constant, and $x_{m}^{*}$ is the closest point to $x$ on the line segment associated with the $m$-th detected direction, $R_{m}$. Note that $I(x ; O)$ is independent of the ordering of the detected wave directions.

To combine information from several sources, there are two immediate ways of incorporating the additional information by either adding or multiplying the functional 
$I^{S}$ evaluated at different observation locations:

$$
\begin{aligned}
& I^{\Sigma}\left(x ; O_{1}, \ldots, O_{N}\right)=\sum_{n=1}^{N} I^{S}\left(x ; O_{n}\right), \\
& I^{\Pi}\left(x ; O_{1}, \ldots, O_{N}\right)=\prod_{n=1}^{N} I^{S}\left(x ; O_{n}\right) .
\end{aligned}
$$

Note again that these two imaging functionals are invariant with respect to the ordering of the observations and detected directions. Such invariance is obviously desirable since the observation and wave direction carry qualitatively similar information. Furthermore, each of these imaging functionals correlates information from different observations, either additively or multiplicative, which is also desirable. However, they both have shortcomings which we will highlight with an example. Suppose that there are two sources and five observation locations as shown in Figure 3.1. In the case of $I^{\Sigma}$, there are many places where at least two rays cross. It is difficult to discern the true sources from artifacts in the image produced by the $I^{\Sigma}$ functional. Additionally, as more observation locations are added, the image does not become sharper. On the other hand, $I^{\Pi}$ produces a sharp image and adding observation locations further sharpens the image. Now, consider the case where one of the observation locations misses a direction, perhaps due to noise or wave cancelation. The $I^{\Sigma}$ image function is robust in such cases, since there will still be many other rays that pass through each source location. Unfortunately, the image produced by $I^{\Pi}$ will not illuminate the location of the missed source, even if new observation locations are added. This example is illustrated in Figure 3.1. To improve the shortcomings of these two ap-
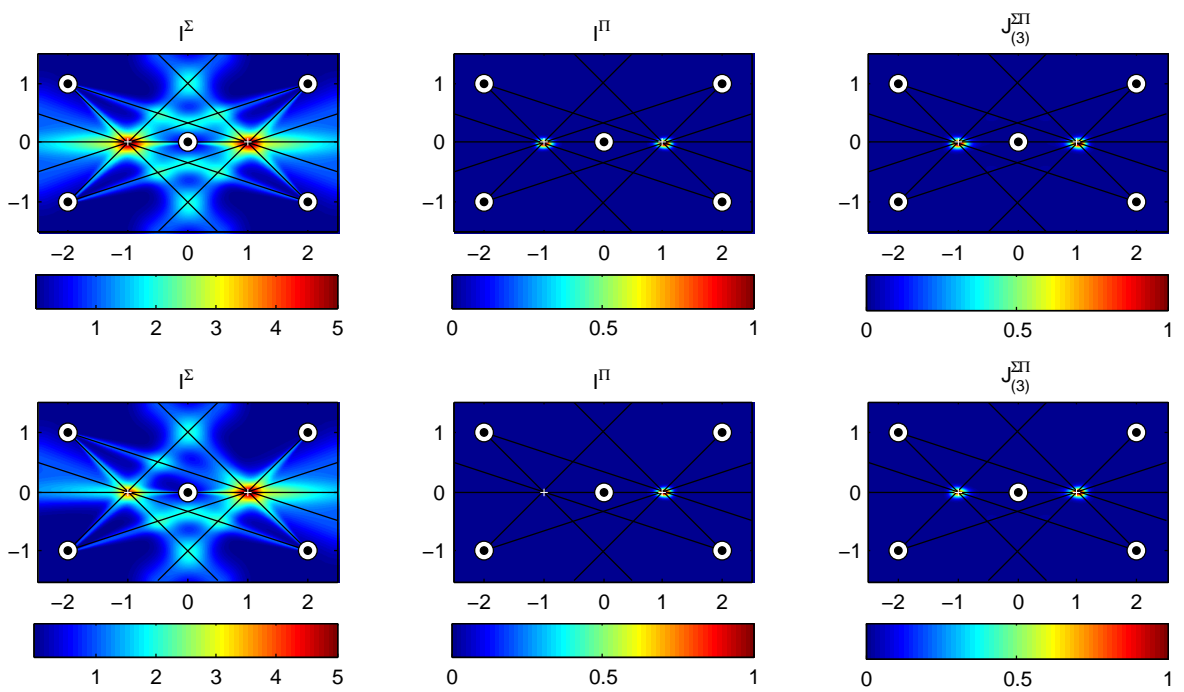

FIG. 3.1. Comparisons of three different imaging functionals. The black lines are backpropagated rays, the observer locations are shown as white dots with a black center and the source locations are indicated by a white cross. The top row of figures shows the ability of $I^{\Pi}$ and $J_{(3)}^{\Sigma \Pi}$ to localize a source when all wave directions are identified at each observation location. The second row of figures shows the effect of missing a direction in the detection step on each of the imaging functionals and the robustness of $J_{(3)}^{\Sigma \Pi}$ in such cases.

proaches, we consider an imaging functional which includes components from both 
$I^{\Sigma}$ and $I^{\Pi}$ :

$$
I_{(3)}^{\Sigma \Pi}\left(x ; O_{1}, \ldots, O_{N}\right)=\sum_{1 \leq i<j<k \leq N} \prod_{q=\{i, j, k\}} I^{S}\left(x ; O_{q}\right) .
$$

That is, we take all combinations of the information from sets of three observation locations, correlate them using $I^{\Pi}$, and sum over all combinations to form the image $I_{(3)}^{\Sigma \Pi}$. Similarly, we could also form image functionals $I_{(n)}^{\Sigma \Pi}$, taking all combinations of the information from sets of $n$ observation locations, correlating them with $I^{\Pi}$, and summing. We specifically use $I_{(3)}^{\Sigma \Pi}$ for $2 \mathrm{D}$, since almost all lines in the plane intersect and this creates many artifacts in the image. For $3 \mathrm{D}$, we expect that $I_{(2)}^{\Sigma \Pi}$ will provide a good image functional.

Finally, we remark that since the solution for the Helmholtz equation decays away from the source and waves travel in straight lines when the index of refraction is constant, we expect the signal from sources behind obstacles or from sources that are far from the observation locations to have very small contribution to the measured data. Thus, in correlating the rays from different observation locations, we will only consider the regions of the domain that are in the intersection of the visibility regions of the considered observation location. Furthermore, we expect that if a source is closer to the observer the likelihood of missing the direction to the source is smaller than the likelihood of missing the direction to a more distant source. In accordance with this, when forming the image at a point $x$, we weight the closer observation locations more heavily. Observation locations immediately next to image points are also weighted less heavily since the plane wave approximation breaks down for sources too close to the observation. We also note that since the imaging functionals above use a sum, we have to carefully rescale the results so that if there are many observations in a particular area of the domain it does not get overly emphasized.

As noted earlier there are many image functionals that satisfy these heuristic rules. In particular, we use the following image functional:

$$
J_{(3)}^{\Sigma \Pi}\left(x ; O_{1}, \ldots, O_{N}\right)=\frac{\sum_{1 \leq i<j<k \leq N} \prod_{q=\{i, j, k\}} H\left(\phi\left(x ; O_{q}\right)\right) \rho\left(\left|x-O_{q}\right|\right)^{\frac{1}{3}} I^{S}\left(x ; O_{q}\right)}{Z_{0}\left(1+\sum_{1 \leq i<j<k \leq N} \prod_{q=\{i, j, k\}} H\left(\phi\left(x ; O_{q}\right)\right) \rho\left(\left|x-O_{q}\right|\right)^{\frac{1}{3}}\right)},
$$

where $H$ is the Heaviside function, $\phi$ is the visibility function (positive in the visible regions, negative in the invisible regions, and zero along the shadow boundary), $Z_{0}$ is a normalization constant so that $J_{(3)}^{\Sigma \Pi} \in[0,1]$ and $\rho(s)=s e^{-\nu_{2} s^{2}}$ with $\nu_{2}$ the localization parameter. In all of the examples, we take $\nu_{2}=1$. The only other parameter is $\nu_{1}$, which appears in the definition of $I^{S}(x ; O)$. We take $\nu_{1}=2 M$, so that is it proportional to the angular discretization which has $M$ points. Again, we design this imaging functional specifically for $2 \mathrm{D}$, by correlating rays from three observation points at a time. However, this imaging functional can be easily modified to correlate the rays from $n$ observation locations at a time by simply taking the sum to be over all possible groups of $n$ observation locations indices, changing the product to include all indices and replacing the $1 / 3$ exponent to be $1 / n$. For example, in $3 \mathrm{D}$, as we expect that correlations of rays from two observation locations will provide good results, we would use the version with $n=2$. 
3.2. Summary of properties of the imaging functional. We summarize the important properties of the imaging functional:

1. Scaling:

- The imaging functional takes on values in $[0,1]$.

- The imaging functional is scaled to account for density of observation locations.

2. Invariance:

- The image is invariant under reordering of detected directions.

- The image is invariant under reordering of observation locations.

3. Correlation: Rays are correlated as sources are likely to be located at intersections of rays.

4. Fault tolerance: The image is not qualitatively changed if a wave direction is missed at an observation location.

5. Localization:

- Rays are only correlated in the intersection of the visibility regions of their associated observation locations.

- Information from closer observation locations is weighted more heavily.

\section{Adaptive algorithm}

In the above section, we have described how to image the sources detected from several observation locations. Below we are going to present an algorithm for choosing the observing locations so that all the sources are detected with a finite number of observations. The algorithm proposed here is derived from the visibility-based environment exploration algorithm introduced in $[37,35]$. The algorithm was originally proposed to explore bounded planar environments with unknown obstacles using the range data to the occluding surfaces. Subsequently, it has been applied to discovering point sources (modeled by elliptic PDEs) in unknown environments [6] and navigation on uneven terrains. Here is the summary of the basic algorithm: 


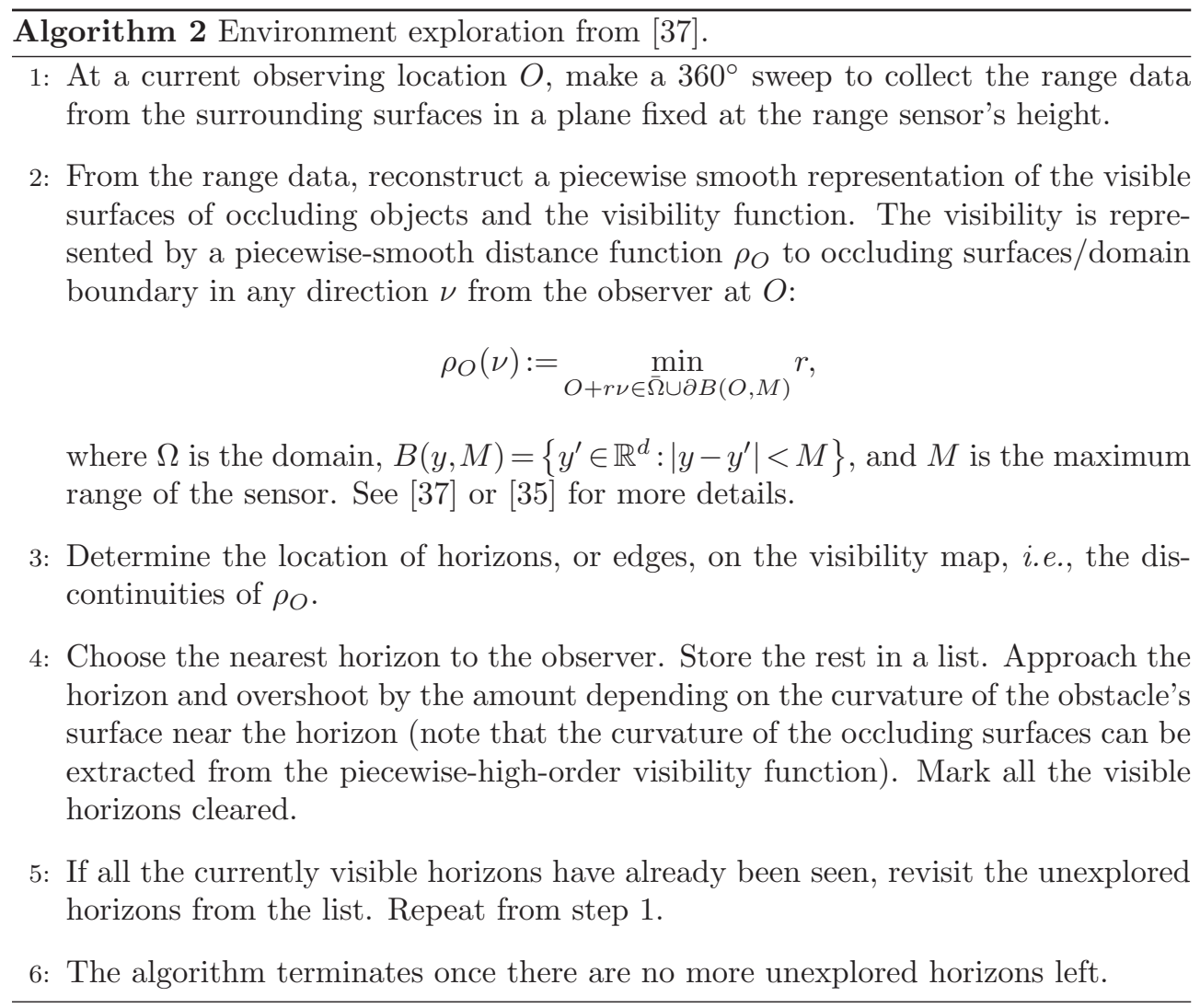

It was shown in [37] that the above algorithm always terminates in a finite number of observations and that the entire bounded domain is explored, i.e. all the obstacles are mapped, at the termination. As a result, a complete map of the environment is reconstructed. In the case where all the obstacles are convex, the maximum number of steps required to explore the entire domain is three times the number of obstacles. This is easy to see if we bound each convex obstacle by a circle. Then, the three vertices of the equilateral triangle surrounding the circle (which are naturally chosen by the above algorithm as observation points) are sufficient to "see" the entire boundary of the obstacle. In practice, however, the number of observations is much smaller than this upper bound [37].

4.1. Discovery of all sources in the domain. We adapt Algorithm 2 for a simultaneous mapping of the environment and discovery of the sources. Based on the imaging functional $J_{(3)}^{\Sigma \Pi}(3.4)$, once a source is detected by at least three local observers (that is, at least three rays intersect at a source point), it appears in the resulting image. Therefore, if every point in the domain outside the obstacles is observed from at least three locations within a certain predefined range, we are guaranteed to detect all the sources in the domain. The proximity of the observers to the source is prescribed by the localization parameter in the imaging functional. This restriction can be easily embedded into the exploration Algorithm 2 by specifying a finite range for the sensor.

Our proposed algorithm simultaneously explores the unknown environment and confirms the candidate sources, which are detected along the way. The observer 
proceeds as in the exploration Algorithm 2, while a small neighborhood around each candidate source is treated as an obstacle, which we will call the possible source regions. In other words, once the imaging function indicates a possible source region, the observer must explore it as if it were a physical obstacle. Correspondingly, these additional obstacles will add new horizons to the list maintained in Algorithm 2. Hence, Algorithm 2 will ensure that the possible source regions are reinspected. Typically, three observations are enough to see the entire boundary of such a region. In order to confirm a source, a ray from each of the three observing positions must pass through the neighborhood in question. As a result of such exploration, some of the candidate sources regions may shift their locations or disappear. Our proposed strategy is described in Algorithm 3 below.

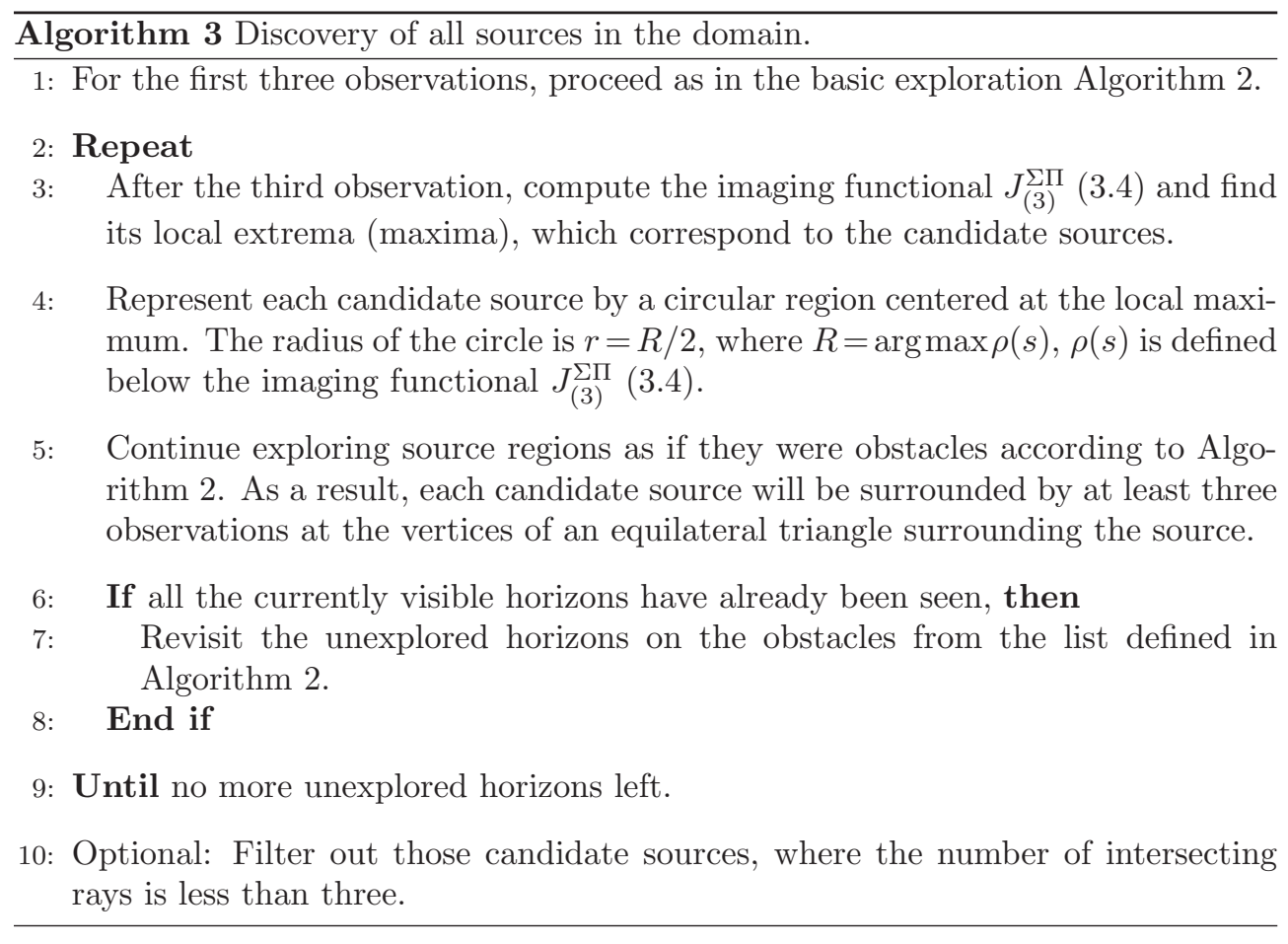

Let us clarify some of the steps of the proposed algorithm. Since the imaging functional $J_{(3)}^{\Sigma \Pi}(3.4)$ is defined for three or more observations, the source detection begins only after the initial three steps of the environment exploration. Once the imaging functional is computed, and we smooth it by convolving with a Gaussian kernel, then the local maxima can be easily identified by thresholding and looking for the points in the image whose values are greater than those of nearest and nextto-nearest neighbors in the image grid. Local extrema which are too close to the obstacles' boundaries are excluded.

In step 4, we construct a circular artificial obstacle around each detected maximum. To explore the resulting source region, the algorithm leads us to place the observer at the vertices of an equilateral triangle surrounding the circle. If the candidate source is the true source, there will be three rays, each coming from a vertex, intersecting at the source location in the center of the circle. As we remarked in the previous section, the likelihood of detecting a source is greater if the source is closer to 
the observer. The localization function $\rho$ in the imaging functional $J_{(3)}^{\Sigma \Pi}(3.4)$ is introduced to emphasize the nearby regions. The radius of the artificial circular obstacle surrounding a candidate source is chosen so that the resulting observing locations are the optimal distance away from the source with respect to the peak of the imaging functional. Note that during the exploration some of the candidate sources may shift their positions or disappear.

Unlike the original exploration algorithm, where the next position is chosen by approaching the nearest horizon, our modified algorithm prioritizes exploration of the candidate sources before continuing with the rest of the environment. Of course, visibility information is accumulated with each step and is used both for obstacle avoidance and the imaging functional updates. Once there are no more unexplored candidate sources, the observer continues mapping the environment and may encounter new sources along the way, for example, if the sources were previously shielded by the obstacles.

The resulting image is used to identify the sources at its local maxima. As a final optional step, we check the number of rays intersecting at each identified source and filter out those sources, where the number of rays is less than three. We further note that our proposed strategy does not miss any of the sources in the domain. However, in cluttered environments a few false sources may be identified.

In an environment with convex disjoint obstacles, the upper bound on the number of observations required to detect all the sources is three times the sum of the number of the obstacles and the number of sources. Experimentally, the number of required observations is much smaller. Note that the complexity of the proposed algorithm, unlike most of the methods in the literature, is independent of the domain discretization and neither the sources nor the observation locations need to be bound to any grid. The algorithm adaptively chooses the next observation location, based on previous observations, and terminates once all the sources have been identified.

4.2. Results. The numerical simulations below are performed at a frequency corresponding to $440 \mathrm{~Hz}$, a reference pitch to which a group of musical instruments are typically tuned for a performance. The sample domain has physical dimensions of $130 \times 130 \mathrm{~m}^{2}$. A few circular scatterers are added to the domain. We assume that every obstacle is at least ten wavelengths in diameter. Even though the piecewisesmooth visibility representation allows for accurate reconstruction of surface geometry and reflecting directions, the reflected secondary and higher order rays are excluded from our source reconstruction procedure due to additional errors introduced during the reconstruction. Sources can be placed anywhere in the environment outside the obstacles.

Figure 4.1 illustrates the setup for our first example. There are seven obstacles (circles) and five sources (crosses). The goal is to detect all the sources while avoiding collisions with the obstacles. The exploration begins at the left bottom corner of the domain. From Figure 4.1(a), one can see a single ray from the initial observer's position passing between the two sources, instead of two rays going through each source. This is the result of wave cancellations due to proximity of the sources, which is inherent in the solution of the Helmholtz equation. Another problem arises in Figure 4.1(b), where the sources appear to be collinear with respect to the second observation location. As a result, a single ray is cast in the direction of both sources. Also note that both rays terminate on the scatterers, from which we can only conclude that the sources are either somewhere along the depicted primary rays or along the secondary reflections, which we are not considering. Furthermore, one 
of the sources on the left should be detectable from this location, however, its ray direction is not detected. During the third observation, depicted in Figure 4.1(c), five rays are detected. While two of the rays seem to pass near or through the sources, the rest are the refections from the numerous surrounding scatterers. The examples

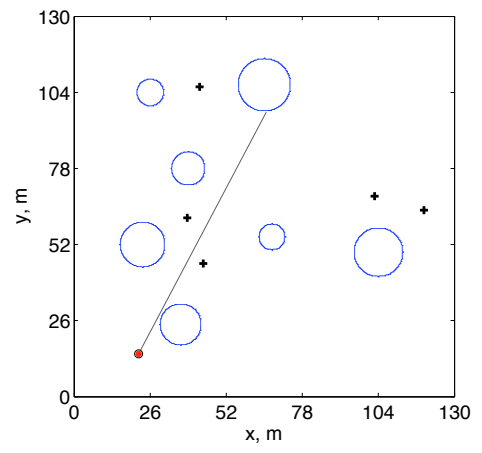

(a) Step 1 .

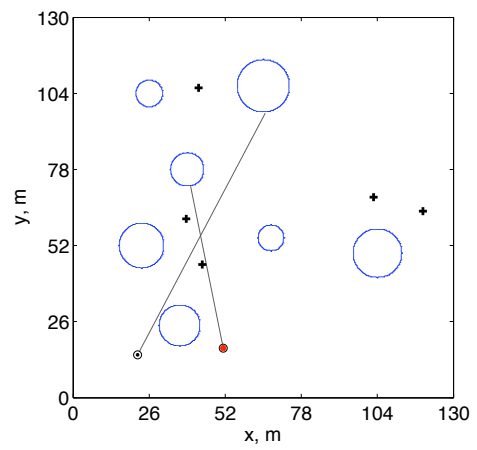

(b) Step 2 .

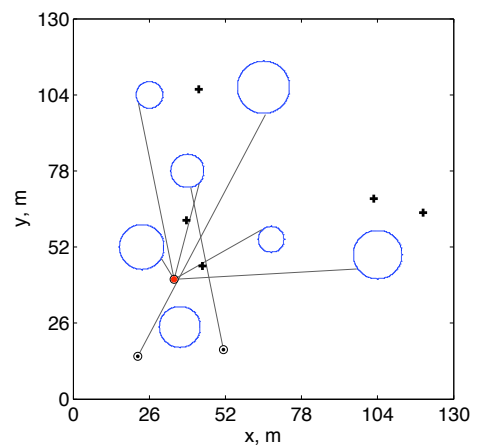

(c) Step 3 .

FIG. 4.1. Example 1: 7 obstacles and 5 sources. The first three steps of the algorithm. Primary rays from each observer's position.

in Figure 4.1 illustrate some of the challenges present in detecting the incoming wave directions in cluttered domains. Our proposed method addresses these challenges by using the imaging functional $J_{(3)}^{\Sigma \Pi}(3.4)$. While the first three steps of the exploration are chosen by the environment mapping algorithm, the imaging functional is computed after the third step, and the observer begins to explore candidate sources. For clarity, we do not show the visibility changes after each step, as it was the subject of previous publications $[37,35]$. Instead, we show the accumulated visible surfaces at each corresponding step of the navigation.

Figure 4.2 contains some key steps of the exploration: the observing locations, the imaging functional, and the estimated sources. The image reconstructed after the first three steps is depicted in Figure 4.2(a). A local maximum is detected and surrounded by a small circular source region. Now the observer must surround the region with at least three observation points. During this process a new maximum is detected (Figure 4.2(b)) and the first candidate source shifts its position toward the true source location, as shown in Figure 4.2(c). After the first seven steps, there are no more candidate source regions left to explore and, therefore, the observer continues by 
exploring the boundary of the nearby obstacle, Figure 4.2(d). New candidate sources appear in Figure 4.2(d) and 4.2(e). In step 11, Figure 4.2(f), one of the candidate sources shifts toward the correct location of the nearby source. Once all the candidate sources on the middle right hand side of the domain have been explored, the observer moves on to explore the rest of the environment in the top left corner, where it encounters a few more sources shown in Figure 4.2(g). At step 20 (Figure 4.2(h)), after one of the candidate source regions is surrounded with three observers, it disappears along with another candidate source region. At this step, exploration of the candidate sources is finished and the remaining three steps complete the environment exploration. The last step of the algorithm is depicted in Figure 4.2(i).

After the exploration is completed, the image of the sums of the rays $I^{\Sigma}$ (3.1) presented in Figure 4.3(a) is analyzed to filter out sources in the regions where the number of passing rays is less than three. As a result, one source (marked by circle), detected during the exploration, is excluded from the detected sources. The final image is depicted on Figure 4.3(b). Note that unlike the image of the sums $I^{\Sigma}(3.1)$, the proposed image functional $J_{(3)}^{\Sigma \Pi}(3.4)$ has very few extrema, which are concentrated around the sources.

The algorithm terminates in 23 steps, while the upper bound for this example is 36 steps ( 7 obstacles plus 5 sources times 3 ). The observation locations are automatically chosen in the vicinity of obstacles and sources, while the empty regions are not unnecessarily observed. Despite the errors and ambiguities associated with detection of incoming rays, illustrated in Figure 4.1, our adaptive sampling algorithm combined with a suitable imaging functional resulted in a reconstruction of the sources and the scatterers.

Our next example is a bit more complicated: there are ten obstacles and seven sources $(\mathrm{d}+)$. The experiment setup is shown in Figure 4.4. We omit the details of the exploration as they are similar to the previous example: candidate sources are detected, then verified. During the exploration, some source regions shift or disappear. Once there are no more new possible sources detected, the observer continues to explore the domain. The exploration terminates in 28 steps, detecting nine sources (marked by $x$ in Figure 4.4(a)). The two extra sources are artifacts caused by the clutter from scattering objects and nearby sources. Filtering based on the number of rays intersecting at the source does not eliminate the spurious sources since there are many rays passing through their neighborhood. In fact, Figure 4.4(b) displays all the primary rays detected from the observation locations. One can see that it is difficult to extract any information about the source locations from just the primary rays. On the other hand, the imaging functional provides concise information about the locations of the sources. Note that the algorithm terminates in 28 steps while the upper bound for this example is 51 steps. The true sources are a subset of the detected sources and one could use an alternative technique to further validate the true sources and exclude the spurious ones.

\section{Conclusion}

In this paper, we studied the inverse problem for the Helmholtz equation in complicated domains with possibly unknown obstacles, which are much larger than the wavelength. We proposed an algorithm which determines the number and the locations of point sources in the domain based on sparse measurements of the wave field. At each observation location the directions of incoming rays are determined from solving a local inverse scattering problem, initially stated in [2]. We formulated the problem as an $L^{1}$ constrained minimization problem and solved it using the Bregman 


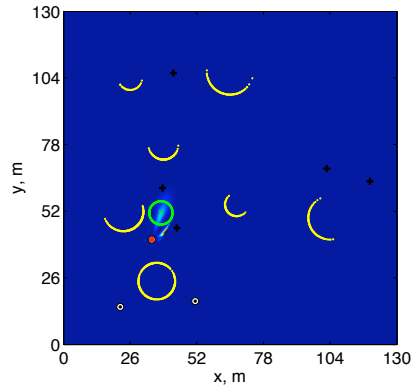

(a) Step3.

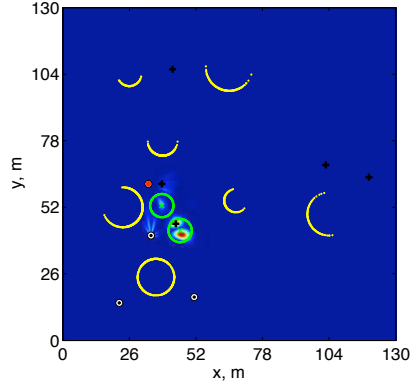

(b) Step4.

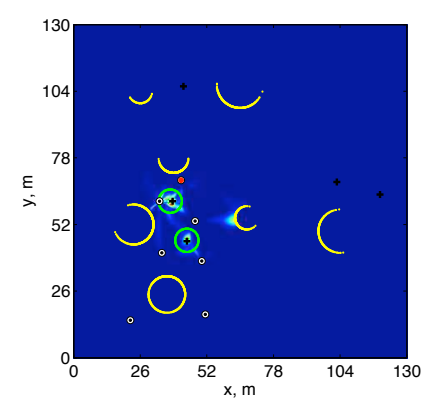

(c) Step7.

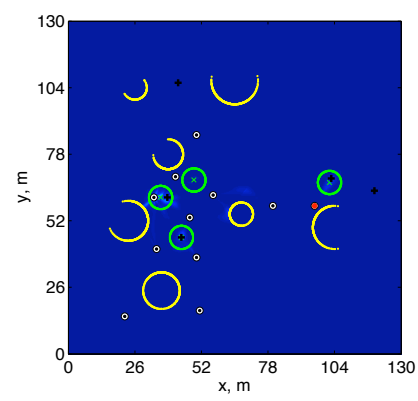

(f) Step 11 .

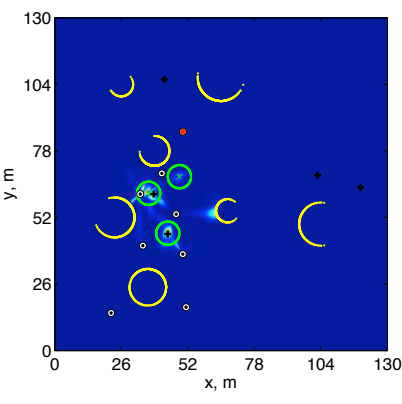

(d) Step 8 .

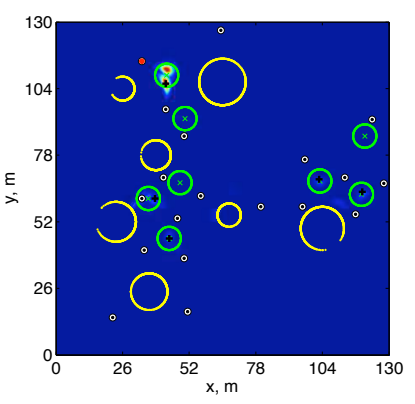

(g) Step 19.

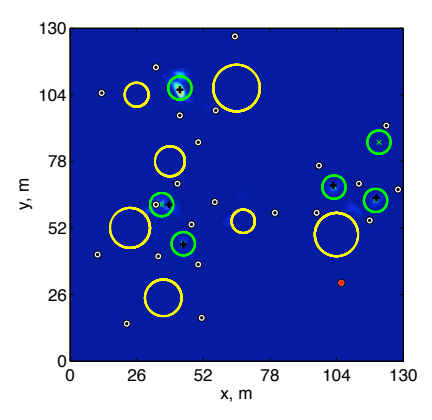

(i) Step 23

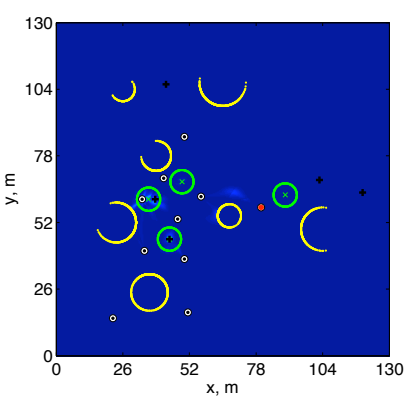

(e) Step 10 .

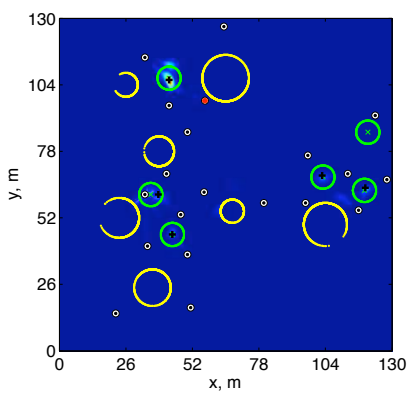

(h) Step 20.

FIG. 4.2. Example 1: 7 obstacles and 5 sources. Some of the steps of the domain exploration and source detection. 


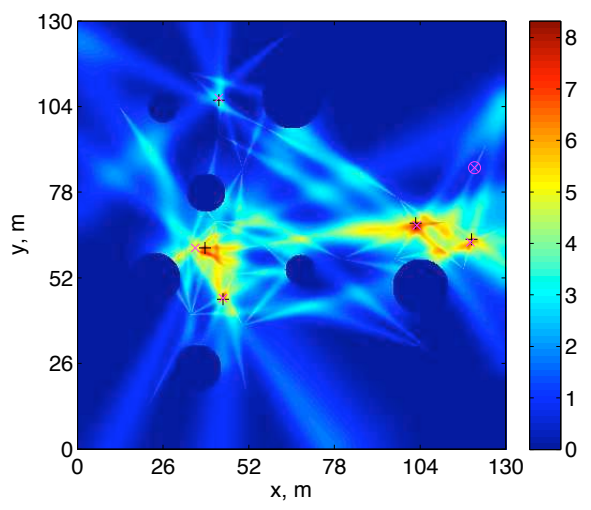

(a) Sums $I^{\Sigma}\left(O_{1}, \ldots, O_{N}\right)$ (3.1). Color bar indicates the number of rays passing through every point of the domain outside the obstacles. The source that has been filtered out is marked by a circle.

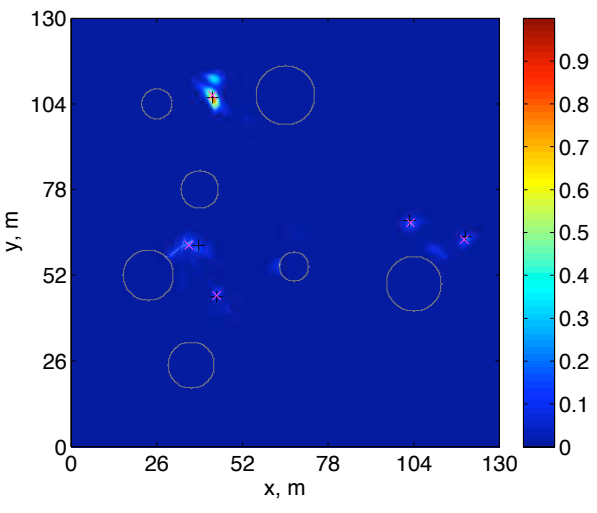

(b) Final image $I_{(3)}^{\Sigma \Pi}\left(O_{1}, \ldots, O_{N}\right)(3.4)$.

FIG. 4.3. Example 1: 7 obstacles and 5 sources. Sums $I^{\Sigma}$ (3.1) and the final image $J_{(3)}^{\Sigma \Pi}$ (3.4). Exact source locations: + , estimated source locations: $\times$.

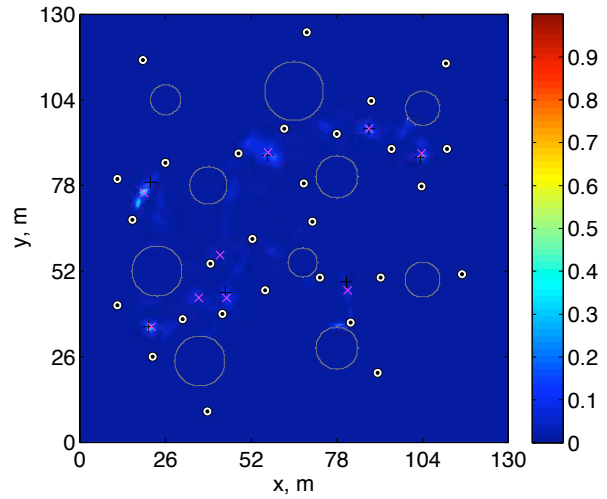

(a) Final image $J_{(3)}^{\Sigma \Pi}\left(O_{1}, \ldots, O_{N}\right)(3.4)$, true $(+)$ and estimated $(\times)$ source locations, 28 observing positions $(\odot)$.

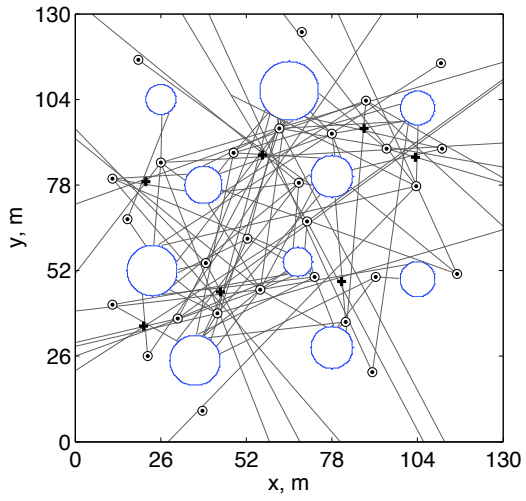

(b) Primary rays at each observing location.

FIG. 4.4. Example 2: 10 obstacles and 7 sources. The final imaging functional $J_{(3)}^{\Sigma \Pi}$ (3.4) with detected sources and the primary rays.

iterative procedure $[47,18]$. The resulting solution is sparse and robust against the noise in the signal measurements. The wave directions can then be traced back to determine the locations of sources at the intersection of rays from several observing positions. The process of detecting ray intersections is prone to instabilities. Instead, we imaged the sources by forming a density functional, which can be thought of as probability density after a renormalization. The resulting image has a few sparse peaks concentrated around the candidate sources.

The observation locations are chosen adaptively based on the imaging functional 
for the previous locations and the visibility information for the domain. The navigation algorithm $[37,35]$ was adapted to this problem, where it has been used to explore unknown environments with obstacles. The algorithm terminates once all the candidate sources and the environment have been explored. To explore a candidate source, the observer must measure the wave field at three locations surrounding the corresponding peak in the imaging functional. As a result, a candidate source may shift its position, disappear, or remain at its current location. In the case when the obstacles are convex, the upper bound on the number of observations needed is three times the sum of the number of obstacles and the number of sources in the domain. However, our numerical simulations indicate that the actual number of steps required to complete the exploration is much smaller than this upper bound. Unlike most methods described in recent literature, the complexity of our proposed strategy is independent of the discretization of the domain for the imaging functional and depends only on the number of sources and obstacles in the domain.

As a result of the exploration, all the sources in the domain are detected and the map of the environment is reconstructed. However, some spurious sources may have also been detected. This happens due to the complex nature of the scattering problem. For example, in a very cluttered environment with many obstacles, the wave field at the measurement location may be complicated by constructive and destructive interference leading to detected rays from many directions. Also, if the size of the scattering obstacles scales in a special way with the wavelength, the resulting wave field may no longer be adequately represented by the local geometric optics approximation. In such cases, alternative formulations (e.g. [30]) must be used.

We finally remark that even though we have presented and validated our algorithm in $2 \mathrm{D}$, the extension to $3 \mathrm{D}$ is not complicated. All of the necessary ingredients, visibility algorithm, incoming ray direction detection and imagining functional can be extended to 3D. For the visibility algorithm the obstacles will be surrounded by a sphere and thus instead of 3 observation locations, one would need 4, all laying on the vertices of a tetrahedron [35]. Once again, the observer will calculate the local curvature and proceed by over-shooting the boundary. The incoming ray direction algorithm is already formulated in [2] based on Legendre polynomials and spherical harmonics. The application of the Bregman iterative methods is direct even in this case. Finally, the imaging function $J_{(3)}^{\Sigma \Pi}$ is dimension independent. As we have remarked earlier, we expect that in 3D one should be able to successfully use the imaging functional $J_{(2)}^{\Sigma \Pi}$, which correlates pair of rays (rather than triples of rays as $J_{(3)}^{\Sigma \Pi}$ ), since $3 \mathrm{D}$ does not suffer from the fact that in $2 \mathrm{D}$ almost all pairs of lines intersect in at least one point.

Acknowledgement. The authors would like to thank Björn Engquist, Stanley Osher and Olof Runborg for insightful conversations.

\section{REFERENCES}

[1] J.D. Benamou, An introduction to Eulerian geometrical optics (1992-2002), J. Sci. Comput., 19(1-3), 63-93, 2003.

[2] J.D. Benamou, F. Collino, and O. Runborg, Numerical microlocal analysis of harmonic wavefields, J. Comput. Phys., 199, 714-741, 2004.

[3] P. Blomgren, G. Papanicolaou, and H. Zhao, Super-resolution in time-reversal acoustics, The Journal of the Acoustical Society of America, 111(1), 230-248, 2002.

[4] L. Borcea, G. Papanicolaou, and C. Tsogka, Imaging and time reversal in random media, Inv. Prob., 18(5), 1247-1279, 2002. 
[5] D.P. Bouche, F.A. Molinet, and R. Mittra, Asymptotic and hybrid techniques for electromagnetic scattering, Proc. of IEEE, 81(12), 1658-1684, 1993.

[6] M. Burger, Y. Landa, N. Tanushev, and R. Tsai, Discovering point sources in unknown environments, in WAFR'08: The 8th International Workshop on the Algorithmic Foundations of Robotics, December, 2008.

[7] J. Cai, S. Osher, and Z. Shen, Linearized Bregman iterations for compressed sensing, Math. Comput., 78, 1515-1536, 2009.

[8] E.J. Candès, The restricted isometry property and its implications for compressed sensing, C. R. Acad. Sci., Paris I, 346, 589-592, 2008.

[9] E.J. Candès, J.K. Romberg, and T. Tao, Robust uncertainty principles: Exact signal reconstruction from highly incomplete frequency information, IEEE Trans. Inform. Theory, 52(2), 489-509, 2006.

[10] E.J. Candès, J.K. Romberg, and T. Tao, Stable signal recovery from incomplete and inaccurate measurements, Commun. Pure Appl. Math., 59(8), 1207-1223, 2006.

[11] E.J. Candès and T. Tao, Decoding by linear programming, IEEE Trans. Inform. Theory, 52, 4203-4215, 2005

[12] E.J. Candès and T. Tao, Near-optimal signal recovery from random projections: Universal encoding strategies? IEEE Trans. Inform. Theory, 52(12), 5406-5425, December, 2006.

[13] S.S. Chen, D.L. Donoho, and M.A. Saunders, Atomic decomposition by basis pursuit, SIAM J. Sci. Comput., 20(1), 33-61, 1998.

[14] Y. Cheng and T. Singh, Source term estimation using convex optimization, in 2008 11th International Conference on Information Fusion, 1-8, 2008.

[15] M. Choulli and M. Yamamoto, Some stability estimates in determining sources and coefficients, J. Inv. Ill-Posed Prob., 14(4), 355-373, 2006.

[16] R. Coifman, V. Rokhlin, and S. Greengard, The fast multipole method for wave equation: A prestrian prescription, IEEE Trans. Antenn. Propag., 35(3), 7-12, 1993.

[17] D. Colton and R. Kress, Inverse Acoustic and Electromagnetic Scattering Theory, Applied Mathematical Sciences, Springer-Verlag, Berlin, second edition, 93, 1998.

[18] J. Darbon and S. Osher, Fast discrete optimizations for sparse approximations and deconvolutions, preprint, 2007.

[19] A. Derode, P. Roux, and M. Fink, Robust acoustic time reversal with High-Order multiple scattering, Physical Review Letters, 75(23):4206, December, 1995.

[20] G. Derveaux, G. Papanicolaou, and C. Tsogka, Time reversal imaging for sensor networks with optimal compensation in time, The Journal of the Acoustical Society of America, 121(4), 2071-2085, April, 2007.

[21] D.L. Donoho, Compressed sensing, IEEE Trans. Inform. Theory, 52(4), 1289-1306, 2006.

[22] A. El-Badia and T. Ha-Duonh, On an inverse problem for the heat equation. Application to a pollution detection problem, J. Inverse Ill-Posed Probl., 10(6), 585-599, 2002.

[23] A. El-Badia, T. Ha-Duonh, and A. Hamdi, Identification of point source in a linear advectiondispersion-reaction equation: Application to a pollution source problem, Inv. Prob., 21(3), 1121-1136, 2005.

[24] B. Engquist and O. Runborg, Computational high frequency wave propagation, Acta Numerica, 12, 181-266, 2003.

[25] A.C. Fannjiang, Compressive inverse scattering: I. High-frequency SIMO/MISO and MIMO measurements, Inv. Prob., 26, 2010.

[26] A.C. Fannjiang, Compressive inverse scattering: II. Multi-shot SISO measurements with born scatterers, Inv. Prob., 26, 2010.

[27] A.C. Fannjiang, K. Solna, and P. Yan, Synthetic aperture imaging of multiple point targets in Rician fading media, SIAM Imag. Sci., 2, 344-366, 2009.

[28] T. Goldstein and S. Osher, The split Bregman method for L1 regularized problems, UCLA CAM Report, (08-92), 2008.

[29] G.H. Golub and C.F. van Loan, Matrix Computations, John Hopkins University Press, 1996.

[30] H.C. Van De Hulst, Multiple Light Scattering. Tables, Formulas, and Applications. Volume 1, Academic Pr, July, 1980.

[31] V. Isakov, Inverse parabolic problems with the final overdetermination, Commun. Pure Appl. Math., 44(2), 185-209, 1991.

[32] F. Claerbout Jon, Fundamentals of Geophysical Data Processing with Application to Petroleum Prospecting, McGraw-Hill, 1976.

[33] J.B. Keller, Geometrical theory of diffraction, J. of the Optical Society of America, 52(2), 116-130, 1962.

[34] V. Komornik and M. Yamamoto, Estimation of point sources and applications to inverse problems, Inv. Prob., 21, 2051-2070, 2005. 
[35] Y. Landa, Visibility of Point Clouds and Exploratory Path Planning in Unknown Environments, $\mathrm{PhD}$ thesis, UCLA, 2008.

[36] Y. Landa, H. Shen, R. Takei, and R. Tsai, Autonomous source discovery and navigation in complicated environments, UCLA, CAM Tech. report 09-73, 2009.

[37] Y. Landa and R. Tsai, Visibility of point clouds and exploratory path planning in unknown environments, Commun. Math. Sci., 6(4), 881-913, 2008.

[38] Y. Landa, R. Tsai, and L.T. Cheng, Visibility of point clouds and mapping of unknown environments, Advanced Concepts for Intelligent Vision Systems, 2006. ACIVS'06, 1014-1025, 2006.

[39] L. Ling, Y.C. Hon, and M. Yamamoto, Inverse source indentification for Poisson equation, Inv. Prob. in Sci. Eng., 13(4), 433-447, 2005.

[40] L. Ling, M. Yamamoto, Y.C. Hon, and T. Takeuchi, Identification of source locations in two dimensional heat equations, Inv. Prob., 22, 1289-1305, 2006.

[41] S. Mallat and Z. Zhang, Matching pursuit in time-frequency dictionary, IEEE Trans. Signal Process., 41(12), 3397-3415, 1993.

[42] S. Osher, M. Burger, D. Goldfarb, J. Xu, and W. Yin, An iterative regularization method for total variation based image restoration, Multiscale Model. Simul., 4(2), 460-489, 2005.

[43] S. Osher, Y. Mao, B. Dong, and W. Yin, Fast linearized Bregman iteration for compressive sensing and sparse denoising, Commun. Math. Sci., 8(1), 93-111, 2010.

[44] Y.C. Pati, R. Rezaiifar, and P.S. Krishnaprasad, Orthogonal matching pursuit: Recursive function approximation with applications to wavelet decomposition, Asilomar Conf. Signals, Systems, and Computers, 27, 1993.

[45] R.O. Schmidt, Multiple emitter location and signal estimation, IEEE Trans. Antenn. Propag., 34, 276-280, 1986

[46] W. Yin, Analysis and generalizations of the linearized Bregman method, Rice Univ, CAAM Tech. report TR09-02, 2009.

[47] W. Yin, S. Osher, D. Goldfarb, and J. Darbon, Bregman iterative algorithms for compressed sensing and related problems, SIAM J. Imag. Sci., 1(1), 143-168, 2008. 